\title{
Бесконечно малые конформные преобразования в римановом пространстве второго приближе- ния
}

\section{Сергей Михайлович Покась}

Аннотация Рассматриваются аналитические конформные преобразования, вводятся и исследуются конформные преобразования второй степени в римановом пространстве второго приближения. Установлена связь между конформными преобразованиями и приближенными конформными преобразованиями.

Ключевые слова риманово пространство, конформные преобразования, обобщенные уравнения Киллинга.

\section{УДК 514.7}

\section{1 Аналитические конформные преобразования}

Рассмотрим риманово пространство $V_{n}$, отнесенное к произвольной системе координат $\left\{x^{1}, x^{2}, \ldots, x^{n}\right\}$. В окрестности любой его фиксированной точки $M_{0}\left(x_{0}^{n}\right)$ построим пространство второго приближения $\widetilde{V}_{n}^{2}\left(y^{n} ; \widetilde{g}_{i j}(y)\right)$, определив его метрический тензор $\widetilde{g}_{i j}(y)$ следующим образом:

$$
\widetilde{g}_{i j}(y)=\underset{\circ}{g_{i j}}+\frac{1}{3} \underset{\circ}{R_{i l_{1} l_{2} j}} y^{l_{1}} y^{l_{2}},
$$

где $g_{i j}=g_{i j}\left(M_{0}\right), \quad R_{i l_{1} l_{2} j}=R_{i l_{1} l_{2} j}\left(M_{0}\right)$.

Подобно тому как в ([3]) изучались аналитические движения в пространстве $\widetilde{V}_{n}^{2}$ будем исследовать аналитические бесконечно малые конформные преобразования: 


$$
y^{\prime h}=y^{h}+t \widetilde{\xi}^{h}(y),
$$

где $\widetilde{\xi}^{h}(y)$ удовлетворяет обобщенным уравнениям Киллинга ([1],[2])

$$
L_{\widetilde{\xi}} \widetilde{g}_{i j}=\frac{\partial \widetilde{\xi}^{\alpha}}{\partial y^{i}} \widetilde{g}_{\alpha j}+\frac{\partial \widetilde{\xi}^{\alpha}}{\partial y^{j}} \widetilde{g}_{\alpha i}+\widetilde{\xi}^{\alpha} \frac{\partial \widetilde{g}_{i j}}{\partial y^{\alpha}}=\psi(y) \widetilde{g}_{i j}
$$

Компоненты вектора смещения $\widetilde{\xi}^{h}(y)$ ищем в виде аналитических функций

$$
\widetilde{\xi}^{h}(y)=\sum_{k=0}^{\infty} a_{k}^{h},
$$

где введено обозначение

$$
a_{k}^{h}=a_{\cdot l_{1} \ldots l_{k}}^{h} y^{l_{1}} \ldots y^{l_{k}} \quad(k=0,1, \ldots) \quad \underset{0}{a^{h}}=a^{h}
$$

В соотношениях (1.5) $a_{.}^{h}, a_{\cdot l_{1}}^{h}, a_{\cdot l_{1} \ldots l_{k}}^{h}$ некоторые постоянные, причем $a_{\cdot l_{1} \ldots l_{k}}^{h} \quad(k \geq 2)$ симметричны по любой паре нижних индексов.

В пространстве $\widetilde{V}_{n}^{2}$ рассмотрим аналитическую функцию п действительных переменных:

$$
\psi(y)=\sum_{k=0}^{\infty} \begin{aligned}
& b \\
& k
\end{aligned}
$$

В (1.6) введены обозначения

$$
\begin{gathered}
b=b_{l_{1} \ldots l_{k}} y^{l_{1}} \ldots y^{l_{k}} \\
b_{k}^{h}=\frac{1}{k+1} \frac{\partial \underset{k+1}{b} g^{\alpha}}{\partial y^{\alpha}} \quad(k=1,2, \ldots)
\end{gathered}
$$

Имеет место утверждение.

Лемма 1 Для того чтобы ряды (1.4) определяли компоненты вектора смещения бесконечно малых конформных преобразований в римановом пространстве второго приближения $\widetilde{V}_{n}^{2}$, необходимо, чтобы члены этих рядов определялись из уравнений и рекуррентных соотношений:

$$
a_{(i j)}=\underset{0}{b} g_{i j}
$$




$$
\begin{gathered}
a_{p}^{h}=-\frac{p-3}{p-1} \underset{p-2}{a_{\alpha}^{\alpha}} t_{\alpha}^{h}+\frac{1}{p}\left(\underset{p-1}{b} y^{h}-\frac{1}{2} \underset{p-2}{b_{0}^{h}} g_{l_{1} l_{2}} y^{l_{1}} y^{l_{2}}\right) \\
(p=2,3, \ldots) \\
t_{k}^{h}=\frac{1}{3} \underset{\circ}{R_{l_{1} l_{2} k}^{h}} y^{l_{1}} y^{l_{2}}
\end{gathered}
$$

Доказательство. Подставим соотношения (1.1), (1.4)-(1.6) в обобщенные уравнения Киллинга (1.3) и приведем подобные члены

$$
\begin{aligned}
& \left(a_{(i j)}-b g_{i j}\right)+\left[2 a_{(i j)}+\frac{1}{3} a^{\alpha} R_{\alpha(i j) l} y^{l}-\underset{1}{b} \underset{\circ}{g_{i j}}\right]+ \\
& +\left\{\begin{array}{c}
3 a_{i j} \\
2
\end{array}+\frac{1}{3}\left[\left(\begin{array}{c}
a_{(i}^{\alpha} R_{j) l_{1} l_{2} \alpha}-b R_{\circ} R_{i l_{1} l_{2} j} \\
\circ
\end{array}\right) y^{l_{1}} y^{l_{2}}+\underset{1}{a_{0}^{\alpha}} R_{\alpha(i j) l} y^{l}\right]-\underset{2}{b} g_{\circ} g_{i j}\right\}+
\end{aligned}
$$

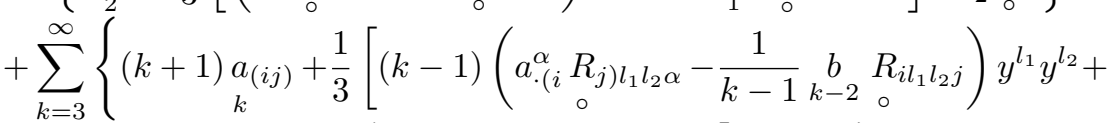

$$
\begin{aligned}
& \left.\left.\left.+\underset{k-2}{a} \underset{\circ}{a} R_{i l_{1} l_{2} j}\right) y^{l_{1}} y^{l_{2}}+\underset{k-2}{a_{\circ}^{\alpha}} R_{\alpha(i j) l} y^{l}\right]-\underset{k}{b} \underset{\circ}{g_{i j}}\right\}=0
\end{aligned}
$$

Так как приведенные коэффициенты при $y^{l_{1}}, y^{l_{2}}, \ldots, y^{l_{n}}$ должны обращаться в нуль, то из (1.10) получаем (1.7), а также

$$
\begin{aligned}
& 2 ! a_{(i j) l_{1}}+\frac{1}{3} a_{.}^{\alpha} \underset{\circ}{R_{\alpha(i j) l_{1}}}-b_{l_{1}} \underset{0}{g_{i j}}=0
\end{aligned}
$$

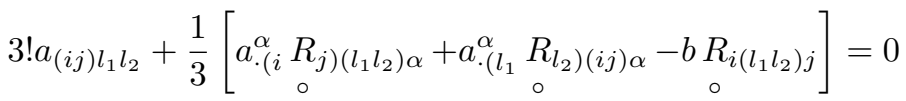

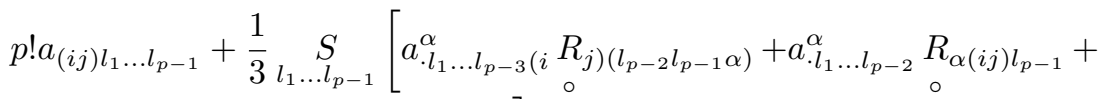

$$
\begin{aligned}
& \left.+b_{l_{1} \ldots l_{p-3}} R_{\circ} R_{i l_{p-2} l_{p-1} j}\right]-(p-1) ! b_{l_{1} \ldots l_{p-1}} g_{\circ} g_{\circ j}=0 \\
& (p=4,5, \ldots)
\end{aligned}
$$

$\left(\underset{l_{1} \ldots l_{p-1}}{S}\right.$ означает операцию симметрирования по индексам $\left.l_{1} \ldots l_{p-1}\right)$.

Уравнения (1.11) проальтернируем по индексам $j$ и $l_{1}$, результат просимметрируем по индексам $i$ и $j$, приняв во внимание уравнения (1.11), результат свернем с $y^{i} y^{j} g_{\circ}^{l_{1} h}$ :

$$
a_{2}^{h}=a^{\alpha} t_{\alpha}^{h}+\frac{1}{2}\left({ }_{1}^{b} y^{h}-\frac{1}{2} b^{h} g_{l_{1} l_{2}} y^{l_{1}} y^{l_{2}}\right)
$$

Поступая аналогичным образом с уравнениями $\left(1.11^{\prime}\right)$, получаем 


$$
\underset{3}{a^{h}}=\frac{1}{3}\left(\underset{2}{b} y^{h}-\frac{1}{2} b_{1}^{h} \underset{\circ}{g_{l_{1} l_{2}}} y^{l_{1}} y^{l_{2}}\right)
$$

Уравнения $\left(1.11^{\prime \prime}\right)$ свернем с $y^{l_{2}} y^{l_{3}} \ldots y^{l_{p-1}}$ и воспользуемся обозначениями $(1.5),(1.6)$ и $(1.9)$ :

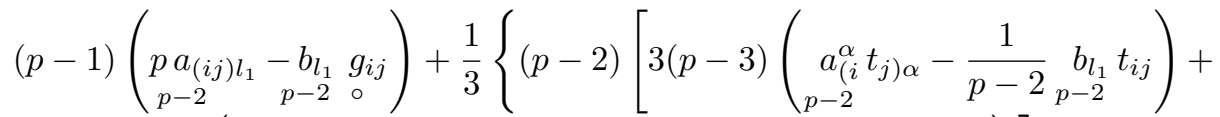

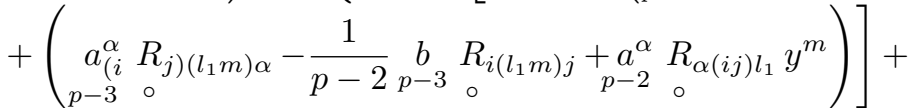

$$
\begin{aligned}
& \left.a_{\cdot l_{1} \ldots l_{p-2}}^{\alpha} R_{\circ(i j) l_{p-1}}\right\}=0
\end{aligned}
$$

Проальтернируем (1.14) по индексам $j$ и $l_{1}$, результат просимметрируем по индексам $i$ и $j$ приняв во внимание уравнения (1.14), получаем:

$$
\begin{aligned}
& 2 p(p-1) a_{l_{1}(i j)}=\frac{1}{3}\left\{( p - 2 ) \left[3(p-3)\left(\begin{array}{c}
-2 a_{i j}^{\alpha} \\
p-2 \\
t_{\alpha l_{1}}-\frac{1}{p-2}
\end{array}\left(\begin{array}{c}
b_{l_{1}} t_{i j}-b_{(i} t_{j) l_{1}} \\
p-2
\end{array}\right)\right)+\right.\right.
\end{aligned}
$$

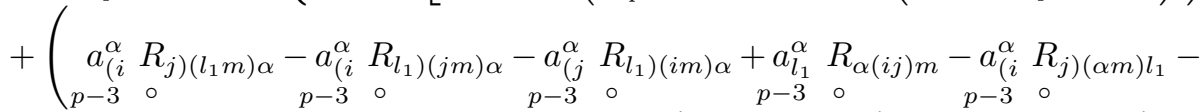

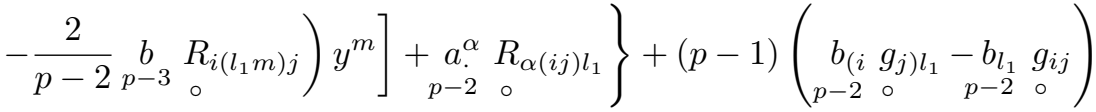

Свернув последнее соотношение с $y^{i} y^{j} g^{l_{1} h}$ и приняв во внимание (1.5), (1.6), (1.9) и алгебраические свойства тензора Римана, получаем (1.8).

Замечание 1 Формулы (1.8) дают выражение $p$-х членов рядов (1.4) через их (р-1)-е члень, обгекты пространства $\tilde{V}_{n}^{2}$ в окрестности точки $M_{0} u$ члень рядов (1.6).

Используя найденные рекуррентные представления, получим формулу общего члена рядов (1.4).

Лемма 2 Для того чтобы рядь (1.4) определяли компоненты вектора смещения аналитических бесконечно малых конформных преобразований в пространстве $\widetilde{V}_{n}^{2}$, необходимо, чтобы $a^{h}$ удовлетворяли уравнениям (1.7), 
а все последующие члены рядов имели вид:

$$
\begin{aligned}
& a_{2}^{h}=a^{\alpha} t_{\alpha}^{h}+\frac{1}{2}\left({ }_{1}^{b} y^{h}-\frac{1}{2} b^{h} g_{l_{1} l_{2}} y^{l_{1}} y^{l_{2}}\right) \\
& a_{2 p}^{h}=\frac{(-1)^{p+1}}{2 p-1} a^{\alpha} t_{\alpha}^{(p) h}+\frac{1}{2 p}\left(\underset{2 p-1}{b} y^{h}-\frac{1}{2} \underset{2 p-2}{b_{\circ}^{h}} g_{l_{1} l_{2}} y^{l_{1}} y^{l_{2}}\right)+ \\
& +\frac{(-1)^{p}}{4(2 p-1)} \sum_{s=1}^{p-1} \frac{2 p-2 s-1}{p-s \quad 2 p-2 s-1} \underset{\alpha}{b^{\alpha}} t^{(s) h} g_{l_{1} l_{2}} y^{l_{1}} y^{l_{2}} \quad(p=2,3, \ldots) \\
& a_{3}^{h}=\frac{1}{3}\left(\underset{2}{b} y^{h}-\frac{1}{2} b_{1}^{h} g_{\circ} g_{l_{1} l_{2}} y^{l_{1}} y^{l_{2}}\right) \\
& a_{5}^{h}=\frac{1}{12} b_{1}^{\alpha} t_{\alpha}^{h} g_{l_{1} l_{2}} y^{l_{1}} y^{l_{2}}+\frac{1}{5}\left(\begin{array}{l}
b \\
4
\end{array} y^{h}-\frac{1}{2} b^{h} g_{l_{1} l_{2}} y^{l_{1}} y^{l_{2}}\right) \\
& \underset{2 p+1}{a_{.}^{h}}=\frac{1}{2 p+1}\left(\underset{2 p}{b} y^{h}-\frac{1}{2} \underset{2 p-1}{b^{h}} g_{\circ}^{g_{l_{1} l_{2}}} y^{l_{1}} y^{l_{2}}\right)+\frac{1}{2 p}\left[\frac{p+1}{2 p+1} 2 p-3 b_{\alpha}^{\alpha} t_{\alpha}^{h}+\right. \\
& \left.+\sum_{s=2}^{p-1} \frac{(-1)^{s+1}(p-s)}{2 p-2 s+12 p-2 s+1} b_{\alpha}^{\alpha} t_{\alpha}^{s(h)}\right]_{l_{l_{1} l_{2}}} y^{l_{1}} y^{l_{2}} \quad(p=3,4, \ldots)
\end{aligned}
$$

Доказательство. Из соотношения (1.8) при $p=2$ имеем

$$
a_{2}^{h}=a^{\alpha} \cdot t_{\alpha}^{h}+\frac{1}{2}\left({ }_{1}^{b} y^{h}-\frac{1}{2} b^{h} g_{l_{1} l_{2}} y^{l_{1}} y^{l_{2}}\right)
$$

Далее из (1.8) при $p=4$ с учетом (1.17) следует, что

$$
a_{4}^{h}=-\frac{1}{3} a^{\alpha} t_{\alpha}^{(2) h}+\frac{1}{3 \cdot 4} b^{h} t_{\alpha}^{h} g_{\circ}^{g_{l_{1} l_{2}}} y^{l_{1}} y^{l_{2}}+\frac{1}{4}\left(\underset{3}{b} y^{h}-\frac{1}{2} \underset{2}{b_{\circ}^{h}} g_{l_{1} l_{2}} y^{l_{1}} y^{l_{2}}\right)
$$

Но к такому же результату мы приходим из (1.15) при $p=2$. Таким образом, формулы (1.15) верны для $p=2$. Пусть они имеют место для $p=m$, т.е.

$$
\begin{aligned}
& \underset{2 m}{a_{.}^{h}}=\frac{(-1)^{m+1}}{2 m-1} a^{\alpha} t_{\alpha}^{(m) h}+\frac{(-1)^{m}}{4(2 m-1)}\left[\frac{2 m-3}{m-12 m-4} b_{\alpha}^{\alpha} t_{\alpha}^{h}+\frac{2 m-5}{m-2} 2 m-6 b^{\alpha} t_{\alpha}^{(2) h}+\right. \\
& \left.+\frac{2 m-7}{m-3} 2 m-5 b_{\alpha}^{\alpha} t^{(3) h}+\ldots+b^{\alpha} t_{\alpha}^{(m-1) h}\right]_{g_{l_{1} l_{2}}} y^{l_{1}} y^{l_{2}}+\frac{1}{2 m}\left(\underset{2 m-1}{b} y^{h}-\frac{1}{2} \underset{2 m-2}{b_{\circ}^{h}} g_{l_{1} l_{2}} y^{l_{1}} y^{l_{2}}\right)
\end{aligned}
$$

Докажем, что она имеет место и для $p=m+1$.

Действительно, из Леммы 1 следует, что

$$
\underset{2 m+2}{a_{\cdot}^{h}}=\left[-\frac{2 m-1}{2 m+12 m} a^{\alpha} t_{\alpha}^{h}+\frac{1}{2 m+2}\left(\underset{2 m+1}{b} y^{h}-\frac{1}{2} \underset{2 m}{b_{\circ}^{h}} \underset{l_{1} l_{2}}{g} y^{l_{1}} y^{l_{2}}\right)\right]
$$

Учитывая (1.19), получаем, что 


$$
\begin{aligned}
& \underset{2 m+2}{a_{\cdot}^{h}}=-\frac{2 m-1}{2 m+1}\left\{\frac{(-1)^{m+1}}{2 m-1} a^{\beta} t_{\beta}^{(m) \alpha}+\frac{(-1)^{(m+1)}}{4(2 m-1)}\left[\frac{2 m-3}{m-1} 2 m-4 b_{\beta}^{\beta} t^{\alpha}+\right.\right. \\
& \left.+\frac{2 m-5}{m-2} b^{\beta} t_{\beta}^{(2) \alpha}++\ldots+b^{\beta} t_{\beta}^{(m-1) \alpha}\right] g_{\circ}^{g_{1} l_{2}} y^{l_{1}} y^{l_{2}}+\frac{1}{2 m}\left(\underset{2 m+1}{b} y^{h}-\right. \\
& \left.\left.-\frac{1}{2} \underset{2 m-2}{b^{\alpha}} g_{l_{1} l_{2}} y^{l_{1}} y^{l_{2}}\right)\right\}+\frac{1}{2 m+2}\left(\underset{2 m+1}{b} y^{h}-\frac{1}{2} \underset{2 m}{b^{h} \underset{\circ}{g_{l_{1} l_{2}}} y^{l_{1}} y^{l_{2}}}\right)
\end{aligned}
$$

Раскрывая скобки и принимая во внимание алгебраические свойства тензора Римана, приходим к (1.15). Аналогично доказывается справедливость формул (1.16).

Исследуем сходимость рядов, члены которых находятся из формул (1.15) и (1.16). Справедливо утверждение.

Лемма 3 Ряды (1.4), члены которых определяются формулами (1.15) и (1.16) сходятся абсолютно и равномерно на множестве

$$
\left|\underset{\circ}{R_{\cdot l_{1} l_{2} k}^{h}} y^{l_{1}} y^{l_{2}}\right| \leq \frac{3 c}{n} \quad(c<1)
$$

Доказательство. Доказательство проведем для (1.15) (для (1.16) доказывается аналогично). Очевидно, что

$$
\begin{aligned}
& \left|\underset{2 p}{a_{.}^{h}}\right| \leq\left|\frac{(-1)^{(p+1)}}{2 p-1} a^{\alpha} \cdot t_{\alpha}^{(p) h}\right|+\left|\frac{1}{2 p} \underset{2 p-1}{b} y^{h}\right|+\frac{1}{4 p}\left|\underset{2 p-2}{b_{\circ}^{h}} g_{l_{1} l_{2}} y^{l_{1}} y^{l_{2}}\right|+ \\
& \frac{1}{4}\left|\frac{(-1)^{p}}{2 p-1} \sum_{s=1}^{p-1} \frac{2 p-2 s-1}{p-s \quad 2 p-2 s-2} \underset{\alpha}{b^{\alpha}} t_{\alpha}^{(s) h}\right| \cdot\left|\underset{\circ}{g_{l_{1} l_{2}}} y^{l_{1}} y^{l_{2}}\right|
\end{aligned}
$$

Оценим каждый модуль отдельно. Введем следующие обозначения:

$$
\max \left\{\left|t_{k}^{h}\right|\right\}=\frac{c}{n}, \quad \max \left\{\left|a^{h}\right|\right\}=c_{1} .
$$

Тогда справедливы следующие оценки:

$$
\begin{gathered}
\left|t_{k}^{(2) h}\right|=\left|t_{\alpha}^{h} \cdot t_{k}^{\alpha}\right| \leq\left|t_{\alpha}^{h}\right| \cdot\left|t_{k}^{\alpha}\right| \leq \frac{c}{n} \cdot \frac{c}{n} \cdot n=\frac{c^{2}}{n} \\
\left|t_{k}^{(3) h}\right| \leq \frac{c^{3}}{n} \\
\cdots \\
\left|t_{k}^{(p) h}\right| \leq \frac{c^{p}}{n}
\end{gathered}
$$

Поэтому 


$$
\left|\frac{(-1)^{(p+1)}}{2 p-1} a^{\alpha} t_{\alpha}^{(p) h}\right| \leq c_{1} \cdot \frac{c^{p}}{n}
$$

Следовательно, ряды $\sum_{p=2}^{\infty} \frac{(-1)^{p}}{2 p-1} a^{\alpha} t_{\alpha}^{(p) h}$ мажорируются числовым рядом $\sum_{p=2}^{\infty} c^{p}$, который сходится при $c<1$. По теореме Вейерштрасса ([5]) указанные ряды сходятся абсолютно и равномерно на множестве

$$
\left|\underset{\circ}{R_{\cdot l_{1} l_{2} k}^{h}} y^{l_{1}} y^{l_{2}}\right| \leq \frac{3 c}{n}
$$

где $c<1$. Далее, ряды $\sum_{p=2}^{\infty} \frac{1}{2 p} \underset{2 p-1}{b}$ и $\sum_{p=2}^{\infty} \frac{1}{p} \underset{2 p-2}{b^{h}}$ сходятся в силу аналитичности функции $\psi(y)$ из (1.6).

Рассмотрим ряды:

$$
\lambda^{h}=\sum_{p=2}^{\infty} \sum_{s=1}^{p-1} \frac{2 p-2 s-1}{2 p-2 s-2} \underset{2 p-2 s-2}{b^{\alpha}} t_{\alpha}^{(s) h}
$$

Нетрудно показать, что (1.20) можно записать в эквивалентном виде:

$$
\lambda^{h}=\sum_{k=0}^{\infty} \frac{2 k+1}{k+1} b^{\alpha} \sum_{s=1}^{\infty} t_{\alpha}^{(s) h} \quad\left(\underset{\circ}{b^{\alpha}}=b^{\alpha}\right)
$$

Поэтому

$$
\begin{gathered}
\left|\sum_{p=2}^{\infty} \sum_{s=1}^{p-1} \frac{2 p-2 s-1}{p-s \quad 2 p-2 s-2} \quad b_{\alpha}^{\alpha}\right|=\left|\sum_{s=1}^{(s) h} t_{\alpha}^{(s) h} \sum_{k=0}^{\infty} \frac{2 k+1}{k+1} b^{\alpha}\right| \leq \\
\leq 2 \sum_{s=1}^{\infty} \sum_{k=0}^{\infty}\left|t^{(s) h}{ }_{2 k}^{\alpha} b^{\alpha}\right|
\end{gathered}
$$

Ho

$$
\left|t_{\alpha}^{(s) h}{ }_{2 k}^{\alpha}\right| \leq\left|t_{\alpha}^{(s) h}\right| \cdot\left|{ }_{2 k}^{b^{\alpha}}\right|=\frac{c^{s}}{n} \sum_{m=1}^{n}\left|\underset{2 k}{b^{m}}\right|
$$

Таким образом,

$$
\sum_{s=1}^{\infty} \sum_{k=0}^{\infty}\left|t^{(s) h}{ }_{2 k}^{b^{\alpha}}\right| \leq \sum_{s=1}^{\infty} \sum_{k=0}^{\infty} \frac{c^{s}}{n}\left(\left|b_{2 k}^{b^{1}}\right|+\left|b_{2 k}^{2}\right|+\ldots+\left|b_{2 k}^{n}\right|\right)=\frac{1}{n} \sum_{s=1}^{\infty} c^{s} \sum_{n=0}^{\infty}\left(\sum_{m=1}^{n}\left|b_{2 k}^{m}\right|\right)
$$


Числовой ряд $\sum_{s=1}^{\infty} c^{s}$ сходится при $c<1$, ряды $\sum_{k=0}^{\infty} \sum_{m=1}^{n}\left|b_{2 k}^{m}\right|$ сходятся в силу аналитичности функции $\psi(y)$ из (1.6). Лемма доказана.

Из уравнений (1.8) и (1.10) имеем:

$$
\begin{aligned}
& \underset{2 p-1}{2 p a_{(i j)}}=\left\{-\frac{2 p-3}{2 p-1}\left[\begin{array}{c}
(2 p-2) a_{(i}^{\alpha} t_{j) \alpha}-a_{\alpha p-2} \mu_{i j}^{\alpha} \\
2 p-3
\end{array}\right]+\right. \\
& +\frac{1}{p}\left[(p-1)\left(\begin{array}{rrr}
b_{(i} & g_{j) l} \\
2 p-2 & y^{l}-b_{i j} & g_{l_{1} l_{2}} \\
2 p-3 & \left.y^{l_{1}} y^{l_{2}}\right)+b_{i j} & g_{i j} \\
2 p-1 & \circ
\end{array}\right]\right\} \\
& \underset{2 p+2}{(2 p+3) a_{(i j)}}=\left\{-\frac{p}{p+1}\left[\begin{array}{c}
(2 p+1) a_{(i}^{\alpha} t_{j) \alpha}-a_{\alpha} \mu_{2 p+1}^{\alpha} \\
2 p
\end{array}\right]+\right. \\
& \left.+\frac{1}{2 p+3}\left[(2 p+1)\left(\begin{array}{rl}
b_{(i} g_{j) l} & y^{l}-b_{i j} g_{l_{1} l_{2}} y^{l_{1}} y^{l_{2}} \\
2 p+1 & \circ
\end{array}\right)+\begin{array}{r}
b_{i j} \\
2 p+2
\end{array} g_{i j}\right]\right\}
\end{aligned}
$$

Подстановка соотношений (1.1), (1.8), (1.10), (1.7) и (1.22) в уравнения (1.8) дает

$$
\begin{aligned}
& a_{(i j)}=\underset{0}{b \underset{i j}{g}} \\
& \frac{4 p}{2 p+1}\left(\begin{array}{c}
a_{(i}^{\alpha} t_{j) \alpha}+a_{2 p} \mu_{i j}^{\alpha} \\
2 p-1
\end{array}\right)+\frac{p}{p+1}\left(\begin{array}{c}
b_{(i} g_{j) l} \\
2 p \text { o }
\end{array}\right. \\
& \left.-b_{i j} g_{l_{1} l_{2}} y^{l_{1}} y^{l_{2}}\right)-\underset{2 p-1}{b} t_{i j}=0 \quad p=1,2, \ldots
\end{aligned}
$$

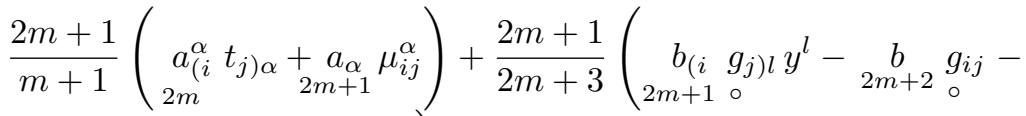

$$
\begin{aligned}
& \left.-b_{i j} g_{l_{1} l_{2}} y^{l_{1}} y^{l_{2}}\right)-\underset{2 m}{b} t_{i j}=0 \quad m=0,1, \ldots
\end{aligned}
$$

Таким образом, доказано утверждение:

Лемма 4 Для того, чтобъ ряды (1.4), членъь которых находятся из рекуррентных соотношений (1.8)-(1.10), определяли компоненты вектора смещения аналитических бесконечно малых конформных преобразований $в$ пространстве $\widetilde{V}_{n}^{2}$, необходимо выполнение уравнений (1.7), (1.23) и (1.24).

Объединяя Леммы 1-4, получаем теорему. 
Теорема 1 Для того, чтобы рлди (1.4) определяли компоненты вектора смещения, аналитических бесконечно малых конформных преобразований в пространстве $\widetilde{V}_{n}^{2}$, необходимо и достаточно выполнение условий (1.7), (1.15), (1.16), (1.23) u (1.24).

Рассмотрим уравнение (1.24) при $m=0$ :

$$
\left.a_{\left(i \underset{\circ}{\alpha} R_{j)\left(l_{1} l_{2}\right) \alpha}+\underset{l_{1} l_{2}}{S}\left[a_{\cdot l_{1}}^{\alpha} \underset{\circ}{R_{\alpha(i j) l_{2}}}+b_{l_{1}(i} g_{\circ} g_{j} l_{2}\right.\right.}-b_{l_{1} l_{2}} g_{\circ} g_{i j}-b_{i j} \underset{\circ}{g_{l_{1} l_{2} i}}-b \underset{\circ}{R_{i l_{1} l_{2} j}}\right]=0
$$

Свертывание последнего соотношения с $g_{\circ} g_{l_{1} l_{2}} g_{\circ}^{i j}$ на основании (1.7) дает

$$
b_{i j} g_{\circ}^{\alpha \beta}=\frac{b R}{2(n-1)}
$$

Свертывая теперь (1.21) с $g_{\circ}^{l_{1} l_{2}} g_{\circ}^{i j}$ и принимая во внимание последнее равенство находим $b_{i j}$ :

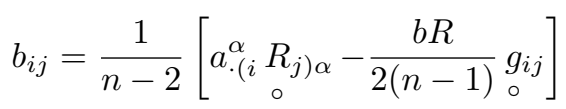

Аналогично поступая с уравнениями (1.23) при $p=1$, используя (1.10), получаем выражение $b_{l_{1} l_{2} l_{3}}$ через $b, b_{l_{1}}, a_{l_{1}}, a_{l_{1} l_{2}}$ и объекты пространства $\widetilde{V}_{n}^{2}$ в точке $M_{0}$. Далее из (1.24) при $m=1$ находим выражение $b_{l_{1} l_{2} l_{3} l_{4}}$ через те же объекты. Продолжая этот процесс, убеждаемся в том, что все члены ряда (1.6) за исключением $b$ и $\underset{1}{b}$ выражаются через $a_{l_{1}}, a_{l_{1} l_{2}}$ и объекты пространства $\widetilde{V}_{n}^{2}$ в точке $M_{0}$.

Замечание 2 Так как члены рлдов (1.4) выражаются через $a^{h}, a_{1}^{h}, b, b$ и обгекты пространства $\widetilde{V}_{n}^{2}$ в точке $M_{0}$, то учитывая уравнения (1.7), (1.23) и (1.24). приходим к известному результату ([4]) о том, что максимальный порядок $r$ группы Ли аналитических бесконечно малых конформных преобразований в римановом пространстве второго приближения $\widetilde{V}_{n}^{2}$ удовлетворяет неравенству

$$
r \leq \frac{(n+1)(n+2)}{2}
$$

\section{2 Конформные преобразования второй степени}

Рассмотрим бесконечно малые конформные преобразования второй степени в пространстве второго приближения $\widetilde{V}_{n}^{2}$ для риманова пространства $V_{n}$, скалярная кривизна $R$ которого в точке $M_{0}$ отлична от нуля. Так как в этом случае в (1.4) 


$$
a_{p}^{h}=0 \quad(p \geq 3)
$$

то из уравнений (1.7) и рекуррентных соотношений (1.8) - (1.10) следует

$$
\begin{aligned}
& a_{(i j)}=\underset{0}{b g_{i j}} \\
& a_{2}^{h}=a^{\alpha} \cdot t_{\alpha}^{h}+\frac{1}{2}\left({ }_{1}^{b} y^{h}-\frac{1}{2} b^{h} g_{\circ} g_{l_{1} l_{2}} y^{l_{1}} y^{l_{2}}\right) \\
& \frac{1}{3} a_{2}^{\alpha} \cdot t_{\alpha}^{h}+\frac{1}{4}\left({ }_{3}^{b} y^{h}-\frac{1}{2}{\underset{2}{2}}_{b_{\circ}}^{h} g_{l_{1} l_{2}} y^{l_{1}} y^{l_{2}}\right)=0 \\
& {\left[\underset{2 m-1}{b} y^{h}-\frac{1}{2} \underset{2 m-2}{b_{\circ}^{h}} g_{l_{1} l_{2}} y^{l_{1}} y^{l_{2}}\right]=0 \quad(m \geq 3)} \\
& {\left[\begin{array}{ccc}
b \\
2 k
\end{array} y^{h}-\frac{1}{2} \underset{2 k-1}{b_{.}^{h}} g_{l_{1} l_{2}} y^{l_{1}} y^{l_{2}}\right]=0 \quad(k=1,2, \ldots)}
\end{aligned}
$$

На основании (2.1) уравнения (1.23) - (1.24) принимают вид:

$$
\begin{aligned}
& \frac{4}{3}\left[a_{(i}^{\alpha} t_{j) \alpha}+\underset{2}{a_{\alpha}} \mu_{i j}^{\alpha}\right]+ \\
& +\frac{1}{2}\left[\underset{\substack{i \\
b_{0}}}{g_{j) l}} y^{l}-\underset{3}{b} \underset{\circ}{g_{i j}}-b_{i j} \underset{\circ}{g_{l_{1} l_{2}}} y^{l_{1}} y^{l_{2}}\right]-\underset{1}{b} t_{i j}=0
\end{aligned}
$$

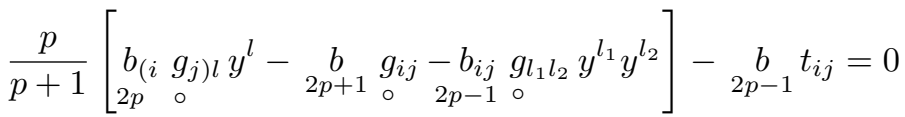

$$
\begin{aligned}
& (p=2,3, \ldots) \\
& {\left[a_{(i}^{\alpha} t_{j) \alpha}+a_{1} \mu_{i j}^{\alpha}\right]+}
\end{aligned}
$$

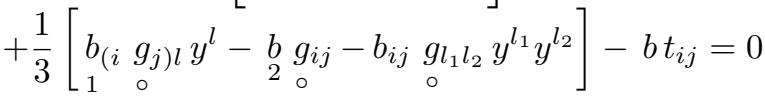

$$
\begin{aligned}
& \frac{2 k+1}{2 k+3}\left[\begin{array}{cc}
b_{(i} & g_{j) l} \\
2 k+1 & \circ
\end{array} y^{l}-\underset{2 k+2}{b} \underset{\circ}{g_{i j}}-b_{2 k} \underset{\circ}{b_{\circ}} g_{l_{1} l_{2}} y^{l_{1}} y^{l_{2}}\right]-\underset{2 k}{b} t_{i j}=0 \\
& (k=1,2, \ldots)
\end{aligned}
$$

Рассмотрим уравнения (2.6). Свернем обе части с $\underset{\circ}{g_{h l}} y^{l}$ :

$$
\left(b_{l_{1} l_{2} \ldots l_{2 k}} g_{l_{2 k+1} l_{2 k+2}}\right) y^{l_{1}} \ldots y^{l_{2 k+2}}=0
$$

Последнее соотношение запишем в эквивалентном виде 


$$
\underset{l_{1} l_{2} \ldots l_{2 k+2}}{S}\left(b_{l_{1} l_{2} \ldots l_{2 k}} g_{\circ} g_{l_{2 k+1} l_{2 k+2}}\right)=0
$$

Свертывание $(2.11)$ с $g_{\circ}^{l_{1} l_{2}} g^{l_{3} l_{4}} \ldots g_{\circ}^{l_{2 k+1} l_{2 k+2}}$ дает:

$$
b_{\alpha_{1} \alpha_{2} \ldots \alpha_{2 k}} g_{\circ}^{\alpha_{1} \alpha_{2}} \ldots g_{\circ}^{\alpha_{2 k-1} \alpha_{2 k}}=0
$$

Свернем теперь (2.11) с $g_{\circ}^{l_{3} l_{4}} \ldots g_{\circ}^{l_{2 k+1} l_{2 k+2}}$ и примем во внимание $(2.12)$ :

$$
b_{l_{1} l_{2} \alpha_{1} \alpha_{2} \ldots \alpha_{2 k-3} \alpha_{2 k-2}} g_{\circ}^{\alpha_{1} \alpha_{2}} \ldots g_{\circ}^{\alpha_{2 k-3} \alpha_{2 k-2}}=0
$$

Свертывание (2.11) с $g_{\circ}^{l_{5} l_{6}} \ldots g^{l_{2 k+1} l_{2 k+2}}$ при условии $(2.13)$ дает

$$
b_{l_{1} l_{2} l_{3} l_{4} \alpha_{1} \alpha_{2} \ldots \alpha_{2 k-5} \alpha_{2 k-4}} g^{\alpha_{0} \alpha_{2}} \ldots g_{\circ}^{\alpha_{2 k-5} \alpha_{2 k-4}}=0
$$

Продолжая этот процесс, на $(k-1)$ шаге мы имеем

$$
b_{l_{1} l_{2} \ldots l_{2 k-2} \alpha \beta} g_{\circ}^{\alpha \beta}=0
$$

И наконец, свернув с $g_{\circ}^{l_{2 k+1} l_{2 k+2}}$ на основании $(2.15)$ убеждаемся в том, что

$$
b_{l_{1} l_{2} \ldots l_{2 k-2}}=0 \quad(k=1,2, \ldots)
$$

Аналогично из (2.5) следует

$$
b_{l_{1} l_{2} \ldots l_{2 m-1}}=0 \quad(m=3,4, \ldots)
$$

Поскльку имеет место (2.16), то все уравнения системы (2.10) выполняются тождественно. Заметим, что согласно (2.17) из сковокупности (2.8) остается только одно - при $p=2$ :

$$
\underset{l_{1} l_{2} \ldots l_{5}}{S}\left(\begin{array}{c}
b_{l_{1} l_{2} l_{3}} \\
R_{\circ} \\
\cos _{4} l_{5}
\end{array}\right)=0
$$

а остальные выполняются тождественно. Из (2.9) на основании (2.16) имеем:

$$
a_{(i}^{\alpha} \underset{\circ}{R_{j)\left(l_{1} l_{2}\right) \alpha}}+a_{\left(l_{1}\right.}^{\alpha} \underset{\circ}{R_{\left.l_{2}\right)(i j) \alpha}}=\underset{\circ}{b} R_{i\left(l_{1} l_{2}\right) j}
$$

Сворачивая последнее уравнение с $g_{\circ}^{l_{1} l_{2}}$, получаем: 


$$
a_{(i}^{\alpha} R_{\circ) \alpha}+a_{\alpha \beta} R_{\cdot(i j)}^{\alpha}=\underset{\circ}{b R_{i j}}
$$

Но из (2.2) вытекает, что

$$
\underset{\alpha \beta}{a_{\alpha} R_{\cdot(i j)}^{\alpha}}=\underset{\quad}{\beta} R_{i j}
$$

поэтому

$$
a_{(i}^{\alpha} \underset{\circ}{\alpha} R_{j) \alpha}=0
$$

Свернем (2.19) с $g^{i j}$, воспользовавшись (2.2) и тем, что скалярная кривизна отлична от нуля, получаем:

$$
b=0
$$

Принимая во внимание соотношения (2.16), (2.17) и (2.20), видим, что функция $\psi(y)$ в (1.6) имеет вид

$$
\psi(y)=b_{l} y^{l}+b_{l_{1} l_{2} l_{3}} y^{l_{1}} y^{l_{2}} y^{l_{3}}
$$

Известно ([1],[2]), решение обобщенных уравнений Киллинга (1.3) при $\psi=$ const определяет вектор смещения бесконечно малых гомотетических преобразований в римановом пространстве. Формула (2.21) свидетельствует о справедливости теоремы.

Теорема 2 Вектор смещения бесконечно малых гомотетических преобразований второй степени в пространстве второго приближения $\widetilde{V}_{n}^{2}$ для $V_{n}$ ненулевой скалярной кривизны в окрестности точки $M_{0}$ по необходимости является вектором Киллинга.

Уравнения (2.9) в соответствии с (2.16) и (2.20) принимают вид

$$
a_{\substack{(i \\ \circ}}^{\alpha} R_{j)\left(l_{1} l_{2}\right) \alpha}+a_{\left(l_{1}\right.}^{\alpha} \underset{\circ}{R_{\left.l_{2}\right)(i j) \alpha}}=0
$$

Далее из уравнений (2.4) имеем

$$
\begin{aligned}
& {\left[\frac { 1 } { 9 } \left(2 a_{j l_{1}}^{\alpha} \underset{\circ}{\left.R_{\alpha l_{2} l_{3} j}+a_{l_{1} l_{2}}^{\alpha} R_{\circ} R_{\alpha\left(j l_{3}\right) i}\right)-}\right.\right.} \\
& \left.-\frac{1}{4}\left(3 b_{j l_{1} l_{2}} g_{\circ} g_{i l_{3}}+b_{l_{1} l_{2} l_{3}} g_{\circ} g_{i j}-b_{i j l_{1}}{\underset{\circ}{\circ}}_{g_{2} l_{3}}-b_{i l_{1} l_{2}}{\underset{\circ}{j}}_{j l_{3}}\right)\right]_{\circ} y^{l_{1}} y^{l_{2}} y^{l_{3}}=0
\end{aligned}
$$


Просимметрировав последнее соотношение по индексам і и j, на основании (2.7) получаем

$$
\begin{gathered}
\left\{a_{l_{1} l_{2}}^{\alpha} R_{\circ} R_{\alpha(i j) l_{3}}-\frac{1}{2} b_{l_{1}} R_{i_{2} l_{3} j}+\right. \\
\left.+\frac{3}{4}\left[3\left(\begin{array}{c}
b_{l_{1} l_{2}(i} g_{j) l_{3}}-b_{i j l_{1}} g_{\circ} g_{l_{2} l_{3}} \\
\circ
\end{array}\right)+b_{l_{1} l_{2} l_{3}} g_{i j}\right]\right\} \mid y^{l_{1}} y^{l_{2}} y^{l_{3}}=0
\end{gathered}
$$

Уравнения (2.23) свернем с $y^{l}$ и результат подставим в (2.4):

$$
\left(\frac{1}{3} b_{l_{1} l_{2} l_{3}} \delta_{l_{4}}^{h}+\frac{1}{2} b_{l_{1} l_{2}}^{h} \underset{\circ}{g_{l_{3} l_{4}}}\right) y^{l_{1}} y^{l_{2}} y^{l_{3}} y^{l_{4}}=0
$$

Из последнего уравнения подобно тому, как получено (2.16), получаем:

$$
b_{l_{1} l_{2} l_{3}}=0
$$

На основании (2.24) уравнения (2.18) (а тогда и все уравнения системы (2.8)) выполняются тождественно.

Подставляя (2.24) в (2.23), получаем:

$$
\begin{gathered}
\left(\underset{l_{1} l_{2}}{a_{\circ}^{\alpha}} R_{\alpha(i j) l_{3}}-\frac{1}{2} b_{l_{1}} R_{\circ} R_{i l_{2} l_{3} j}\right) y^{l_{1}} y^{l_{2}} y^{l_{3}}=0 \\
\left(\underset{l_{1} l_{2}}{a_{\circ}^{\alpha} R_{l_{3} l_{4} \alpha}^{h}}\right) y^{l_{1}} y^{l_{2}} y^{l_{3}} y^{l_{4}}=0
\end{gathered}
$$

Легко видеть, что уравнения (2.26) (а тогда и уравнения (2.4)) выполняются тождественно вследствие (2.25).

Продифференцировав $(2.26)$ по $y^{i}$, опустив $h$ с помощью $g_{h}$, результат просимметрируем по индексам $i$ и $j$ и учтем (2.25):

$$
\left(a_{l_{1}(i}^{\alpha} \underset{\circ}{R_{j) l_{2} l_{3} \alpha}}-\frac{1}{4} b_{l_{1}} R_{\circ} R_{i l_{2} l_{3} j}\right) y^{l_{1}} y^{l_{2}} y^{l_{3}}=0
$$

Тогда очевидно, что уравнения (2.7) есть не что иное, как сумма уравнений (2.25) и (2.27) при условии (2.24).

Подстановка $(2.3)$ в (2.25) и (2.24) в (2.21) дает соответственно

$$
\begin{aligned}
& \underset{l_{1} l_{2} l_{3}}{C}\left[\begin{array}{cc}
a_{\cdot}^{\alpha} R_{\alpha\left(l_{1} l_{2}\right) \beta} & R_{\cdot(i j) l_{3}}^{\beta}
\end{array}-\frac{3}{2}\left(\begin{array}{c}
b_{\alpha} R_{\cdot(i j) l_{1}}^{\alpha} \underset{\circ}{g_{l_{2} l_{3}}}-b_{l_{1}} R_{\circ\left(l_{2} l_{3}\right) j} \\
\circ
\end{array}\right)\right]=0 \\
& \psi(y)=b_{l} y^{l}
\end{aligned}
$$

В результате проведенных рассуждений приходим к теореме 
Теорема 3 В пространстве второго приближения $\widetilde{V}_{n}^{2}$ для риманова пространства $V_{n}$ ненулевой скалярной кривизны в точке $M_{0}$ существуют бесконечно малое конформное преобразование второй степени вида

$$
\widetilde{\xi}^{h}=a_{.}^{h}+a_{\cdot l}^{h} y^{l}+a_{\cdot l_{1} l_{2}}^{h} y^{l_{1}} y^{l_{2}},
$$

отличное от движения тогда и только тогда, когда

$$
\begin{aligned}
& a_{(i j)}=0
\end{aligned}
$$

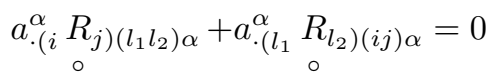

$$
\begin{aligned}
& a_{2}^{h}=a^{\alpha} \cdot t_{\alpha}^{h}+\frac{1}{2}\left({ }_{1}^{b} y^{h}-\frac{1}{2} b^{h} g_{\circ} l_{l_{1} l_{2}} y^{l_{1}} y^{l_{2}}\right) \\
& \underset{l_{1} l_{2} l_{3}}{C}\left[\begin{array}{cc}
a_{\cdot}^{\alpha} R_{\alpha\left(l_{1} l_{2}\right) \beta} & R_{\cdot(i j) l_{3}}^{\beta} \\
\circ & -\frac{3}{2}
\end{array}\left(\begin{array}{c}
b_{\alpha} R_{\cdot(i j) l_{1}}^{\alpha} \underset{\circ}{g_{l_{2} l_{3}}}-b_{l_{1}} \\
R_{\circ\left(l_{2} l_{3}\right) j}
\end{array}\right)\right]=0 \\
& \psi(y)=b_{l} y^{l} \quad\left(b_{l} \neq 0\right)
\end{aligned}
$$

\section{3 Приближенные конформные преобразования}

Подобно тому, как вводилось понятие приближенного вектора Киллинга ([3]) определяется приближенный обобщенный вектор Киллинга.

Определение Вектор $\xi^{h}(x)$ называется приближенным конформным вектором Киллинга второго порядка в окрестности точки $M_{0}$ риманова пространства $V_{n}$, если для него в этой точке выполняются обобщенные уравнения Киллинга и их ковариантные дифберенцированное продолжение первого порядка.

Бесконечно малое преобразование риманова пространства $V_{n}$, которое порождается приближенным конформным вектором Киллинга второго порядка, называется приближенным бесконечно малым конформным преобразованием второго порядка.

То аналогично с Теоремой 8 ([3]) доказываются утверждения.

Теорема 4 Приближенный конформный вектор Киллинга $\xi^{h}(x)$ риманова пространства $V_{n}$ ненулевой скалярной кривизны $R$ в точке $M_{0}$ определяется в пространстве второго приближения $\widetilde{V}_{n}^{2}$ вектора второй степени $\widetilde{\xi}^{h}(y)$, который является приближенным обобщенным вектором Киллинга второго порядка в окрестности точки $M_{0}$. 
Теорема 5 Бесконечно малое конформное преобразование второй степени в пространстве второго приближения $\widetilde{V}_{n}^{2}$ для риманова пространства $V_{n}$ ненулевой скалярной кривизны $R$ в точке $M_{0}$ реализует приближенные бесконечно малое конформное преобразование второго порлдка в окрестности точки $M_{0}$ исходного $V_{n}$, отнесенного к римановой системе координат с началом в этой точке.

\section{Список литературы}

1. Эйзенхарт Л.П.: Риманова геометрия. М, ИЛ, 1948, 316 стр.

2. Эйзенхарт Л.П.: Непрерывные группы преобразований. М, ИЛ, 1947, 287 стр.

3. Покась C.М.: Группы Ли движений в римановом пространстве второго приближения. Известия Пензенского государственного университета имени В.Г. Белинского, физико-математические науки, №26, 2011, стр. 173-183

4. Петров А.З.: Новые методы в теории относительности. М, Наука, 1966, 495 стр.

5. Будак Б.М., Фомин С.В.: Краткие интегралы и ряды. М, Наука, 1967, 607 стр.

\section{Сергей Михайлович Покась}

Одесский национальный университет имени И. И. Мечникова, кафедра геометрии и топологии, Одесса, Украина

E-mail: pokas@onu.edu.ua

\section{Sergey M. Pokas}

\section{Infinitesimal conformal transformations in a Riemannian space of the second approximation}

We discuss analytic conformal transformations, introduce and examine second degree conformal movements in a Riemannian space of the second approximation. Established a connection between conformal movements and approximate conformal movements in spaces $V_{n}$ and $\widetilde{V}_{n}^{2}$. 